CERN-TH-97-347

RAL-TR-97-062

IOA-TH-97-017

Ref. SISSA 155/97/EP

hep-ph/9712446

\title{
Hybrid MSW + VO Solution of the Solar Neutrino Problem in String-Motivated Unified Theories
}

\author{
B. C. Allanach ${ }^{1}$, G. K. Leontaris ${ }^{2,3}$ and S. T. Petcov ${ }^{4,5}$ \\ 1. Rutherford Appleton Laboratory, Chilton, Didcot, Oxon. OX11 0QX, \\ United Kingdom \\ 2. CERN Theory Division, 1211 Geneva 23, Switzerland \\ 3. Physics Department, University of Ionnanina, Ioannina, GR-45110, Greece \\ 4. Scuola Internazionale Superiore di Studi Avanzati (SISSA), and Istituto Nazionale \\ di Fisica Nucleare, I-34013, Trieste, Italy \\ 5. Institute of Nuclear Research and Nuclear Energy, Bulgarian Academy of Sciences, \\ 1784 Sofia, Bulgaria
}

\begin{abstract}
It is shown that the hybrid MSW + VO solution of the solar neutrino problem, according to which the solar $\nu_{e}$ undergo matter-enhanced transitions into $\nu_{\mu, \tau}$ in the Sun followed by long wave length $\left(\sim 1.5 \times 10^{8} \mathrm{~km}\right) \nu_{e} \leftrightarrow \nu_{\mu, \tau}$ oscillations in vacuum between the Sun and the Earth, can occur naturally in string-motivated grand unified theories. We consider the supersymmetric version of a string-type $S U(4) \otimes S U(2)_{L} \otimes S U(2)_{R}$ theory with $U(1)_{X}$ family symmetry, which was shown to successfully describe the charged fermion masses and the quark mixing, and extend the earlier fermion mass analysis to the neutrino sector. We show that the four oscillation parameters $\Delta m_{31}^{2}$, $\Delta m_{21}^{2}$ and $\sin ^{2} 2 \theta_{12}, \sin ^{2} 2 \theta_{13}$, characterising the combined matter-enhanced transitions and vacuum oscillations of the solar $\nu_{e}$, naturally get values in the ranges of the hybrid $\mathrm{MSW}+\mathrm{VO}$ solutions found recently.
\end{abstract}




\section{Introduction}

In the present article we show that the hybrid matter-enhanced transitions + vacuum oscillations solutions of the solar neutrino problem, possible in the case of three flavour neutrino mixing and found in ref. [1], can appear naturally in a class of string-type unified theories. As is well-known, the solar neutrino observations provide strong evidences for the existence of non-zero neutrino masses and lepton mixing. The signals measured in all six experiments which have obtained data on the solar neutrino flux so far, Homestake, Kamiokande II and III, SAGE, GALLEX and Super-Kamiokande, are considerably smaller than the signals predicted by the standard solar models. The case for a significant solar neutrino deficit was reinforced recently [2] with the publication of new more precise helioseismological data (see [2] for the relevant references). These data made possible to perform a number of critical tests of the solar models, including the models which have been constructed with the purpose of solving the solar neutrino problem without invoking "unconventional" neutrino behaviour (matterenhanced transitions, vacuum oscillations, etc.). The analyses completed so far showed [2] that only the standard solar models which include the diffusion of heavy elements in the solar interior are compatible with the most recent helioseismological observations, and that none of these models can describe the current solar neutrino data. The indicated results make the possibility of an "unconventional" behaviour of the solar $\nu_{e}$ on their way from the central part of the Sun to the Earth surface look at present more plausible than ever in the past. However, the crucial unambiguous experimental proof for such a behaviour is still lacking and it is hoped that the presently operating and the future solar neutrino experiments will be able to determine the true cause of the solar neutrino problem.

The hybrid resonance transitions + vacuum oscillations (MSW + VO) solution is based on the assumption that the solar $\nu_{e}$ undergo matter-enhanced transitions into $\nu_{\mu}$ and/or $\nu_{\tau}$ when they propagate from the central part to the surface of the Sun and that these transitions are followed by long wave length $\left(\sim 1.5 \times 10^{8} \mathrm{~km}\right)$ vacuum oscillations of the $\nu_{e}$ and/or $\nu_{\mu(\tau)}$ when the flavour neutrinos travel from the surface of the Sun to the Earth. The solution of interest is a genuine three-flavour-neutrino mixing solution since it is possible only if the three flavour neutrinos mix in vacuum:

$$
\left|\nu_{l}>=\sum_{k=1}^{3} U_{l k}^{*}\right| \nu_{k}>, \quad l=e, \mu, \tau,
$$

where $\mid \nu_{l}>$ is the state vector of the (left-handed) flavour neutrino $\nu_{l}$ having a definite 
momentum $(\vec{p}), \mid \nu_{k}>$ is the state vector of a neutrino $\nu_{k}$ possessing a definite mass $m_{k}$ and a definite momentum $(\vec{p}), m_{k} \neq m_{j}, k \neq j=1,2,3, m_{1}<m_{2}<m_{3}$ 円 , and $U$ is a $3 \times 3$ unitary matrix - the lepton mixing matrix. The (average) solar $\nu_{e}$ survival probability, $\bar{P}\left(\nu_{e} \rightarrow \nu_{e}\right)$, which is a basic quantity in the analysis of the solar neutrino data in the case of interest and for which a simple analytic expression was derived in [1], depends on four neutrino mass and mixing parameters: two basically responsible for the matter-enhanced transitions and another two responsible for the long wave length vacuum oscillations. In view of our further discussion we will assume these parameters to be respectively $\Delta m_{31}^{2}, \sin ^{2} 2 \theta_{13}$ and $\Delta m_{21}^{2}, \sin ^{2} 2 \theta_{12}$, where as usual $\Delta m_{i j}^{2}=m_{i}^{2}-m_{j}^{2}$, and

$$
\begin{gathered}
\cos 2 \theta_{13}=1-2\left|U_{e 3}\right|^{2}>0, \quad \sin 2 \theta_{13}=2\left|U_{e 3}\right| \sqrt{1-\left|U_{e 3}\right|^{2}}>0 \\
\sin ^{2} 2 \theta_{12}=4 \frac{\left|U_{e 1}\right|^{2}\left|U_{e 2}\right|^{2}}{\left(\left|U_{e 1}\right|^{2}+\left|U_{e 2}\right|^{2}\right)^{2}}
\end{gathered}
$$

As was shown in ref. [1] on the basis of an analysis of the recent solar neutrino data, the MSW + VO solution is possible for

$$
\begin{aligned}
4.0 \times 10^{-12} e V^{2} & \lesssim \Delta m_{21}^{2} \lesssim 5.0 \times 10^{-10} e V^{2}, \\
0.15 & \lesssim \sin ^{2} 2 \theta_{12} \leq 1.0
\end{aligned}
$$

and

$$
\begin{gathered}
5.0 \times 10^{-6} \mathrm{eV}^{2} \lesssim \Delta m_{31}^{2} \lesssim 1.5 \times 10^{-4} \mathrm{eV}^{2}, \\
3.1 \times 10^{-4} \lesssim \sin ^{2} 2 \theta_{13} \lesssim 0.5 .
\end{gathered}
$$

More specifically, in [1] five different sub-regions in the region of the space of parameters, defined by eqs. (2) - (7), corresponding to five different types of hybrid MSW + VO solutions, A, B, C, D, and E, have been identified (see Figs. 1 a-11c). Let us add that, what actually specifies the solutions $A, B, C, D$ and $E$ and makes them physically very different is the distinct way the various solar neutrino flux components $\left(p p,{ }^{7} \mathrm{Be},{ }^{8} \mathrm{~B}\right.$, etc.) are affected by the matter-enhanced transitions and the vacuum oscillations in the case of each particular solution (see [1] for further details).

As it follows from eqs. (2) - (7), the combined MSW + VO solution requires rather small neutrino mass squared differences and very specific mixing in the lepton sector and ratio of $\Delta m_{21}^{2}$ and $\Delta m_{31}^{2}$. In the context of GUT's, the light neutrino masses can be generated, as is well-known, by the see-saw mechanism: $m_{\nu} \sim m_{Q}^{2} / M_{G U T}$, where $m_{Q}$ is

\footnotetext{
${ }^{1}$ It is always possible to choose $m_{1}<m_{2}<m_{3}$ without loss of generality and we will work with this convention in what follows.
} 
the up quark mass and $M_{G U T}$ is the unification scale. Moreover, making use of simple discrete or continuous $U(1)$ symmetries at the unification scale, specific textures for the fermion mass matrices can be constructed with a minimum number of parameters which leads, in particular, to rather specific predictions for the mixing in the lepton sector. Effective unified or partially unified GUTs which incorporate naturally the indicated properties can be built within the string theories. In the free fermionic formulationfor

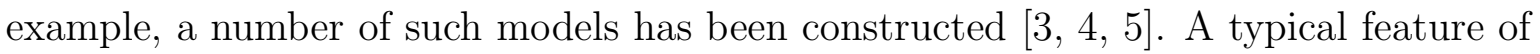
the latter is that in addition to the gauge group containing the $\mathrm{SU}(3) \otimes \mathrm{SU}(2)_{L} \otimes \mathrm{U}(1)_{Y}$ as a subgroup, a number of surplus $U(1)$ symmetry factors which distinguish between the three fermion families appear. However, models based on these constructions do not contain Higgs fields in the adjoint or higher dimensional representations. As a consequence, the unification symmetry of the traditional GUT's like $\mathrm{SU}(5)$ and $\mathrm{SO}(10)$, cannot be broken down to the symmetry of the Standard Theory.

Attempts to overcome this difficulty led to constructions of partially unified GUT's in which only small-dimensional Higgs representations are needed to break the unification symmetry [3, 国. A partially unified theory which fulfils all basic requirements for a string-type GUT [4], is based on the Pati-Salam [6] gauge symmetry group $\mathrm{SU}(4) \otimes \mathrm{O}(4)$. The $\mathrm{SU}(4) \otimes \mathrm{O}(4)$ unification symmetry can be broken down 田 to the standard model gauge symmetry without using adjoint or any higher dimensional Higgs representations. The model possesses a number of attractive features. Colour triplets and Higgs doublets arise in different representations. Thus, it is free from doublet-triplet splitting complications as the triplets become massive from simple trilinear couplings. There are no dangerous proton decay mediating gauge bosons. As a consequence, the $\mathrm{SU}(4) \otimes \mathrm{O}(4)$ breaking scale $M_{G U T}$ can in principle be lower than the symmetry breaking scale of other unified theories, (e.g., the $\mathrm{SO}(10)$ theory). In fact, the basic constraints on $M_{G U T}$ are the low energy measurements of the strong coupling constant $\alpha_{s}$ and of $\sin ^{2} \theta_{W}$. Thus, in the case when only the minimal supersymmetric standard model (MSSM) spectrum appears below $M_{G U T}$ these parameters are correctly predicted if $M_{G U T} \sim 10^{16} \mathrm{GeV}$. Furthermore, a recent non-renormalisable operator analysis [7] of the model's supersymmetric version has revealed quite remarkable features of the charged fermion mass matrices.

In this work we demonstrate that the hybrid MSW + VO scenario, shown recently to provide an alternative solution of the solar neutrino problem [1] (see also, e.g., [8]), can be naturally implemented in the string-type partially unified $\mathrm{SU}(4) \otimes \mathrm{O}(4)$ theory. Our analysis will be performed in the context of the supersymmetric version of the 
theory with the $\mathrm{SU}(4) \otimes \mathrm{O}(4)$ gauge symmetry augmented by a $U(1)$ family symmetry.

\section{The $\mathrm{SU}(4) \otimes \mathrm{O}(4)$ Model}

Here we briefly summarise the basic features of the model which are relevant for our analysis [7]. The gauge group is $\mathrm{SU}(4) \otimes \mathrm{O}(4) \otimes \mathrm{U}(1)_{X}$, or equivalently its isomorphic (enhanced) Pati-Salam symmetry

$$
\mathrm{SU}(4) \otimes \mathrm{SU}(2)_{L} \otimes \mathrm{SU}(2)_{R} \otimes \mathrm{U}(1)_{X}
$$

where $\mathrm{U}(1)_{X}$ is a gauged family symmetry of the same type as discussed in ref. [9]. The left- and right-handed fermion fields are accommodated in the following representations,

$$
\begin{aligned}
F^{i^{\alpha a}} & =(4,2,1)=\left(\begin{array}{llll}
u^{R} & u^{B} & u^{G} & \nu \\
d^{R} & d^{B} & d^{G} & e^{-}
\end{array}\right)^{i} \\
\bar{F}_{x \alpha}^{i} & =(\overline{4}, 1, \overline{2})=\left(\begin{array}{cccc}
\bar{d}^{R} & \bar{d}^{B} & \bar{d}^{G} & e^{+} \\
\bar{u}^{R} & \bar{u}^{B} & \bar{u}^{G} & \bar{\nu}
\end{array}\right)^{i}
\end{aligned}
$$

where $\alpha=1, \ldots, 4$ is an $\mathrm{SU}(4)$ index, $a, x=1,2$ are $\mathrm{SU}(2)_{L, R}$ indices, and $i=1,2,3$ is a family index. The $\mathrm{U}(1)_{X}$ family symmetry is broken spontaneously below the string scale $M_{S}$ by the superfields $\theta, \bar{\theta}$, both singlets under the $\mathrm{SU}(4) \otimes \mathrm{SU}(2)_{L} \otimes$ $\mathrm{SU}(2)_{R}$ symmetry, but with $\mathrm{U}(1)_{X}$ charges $+1,-1$ respectively. This breaking cancels anomalous D-terms provided the vacuum expectation values of $\theta$ and $\bar{\theta}$ satisfy 8 []]

$$
\langle\theta\rangle \sim\langle\bar{\theta}\rangle \sim(0.1-0.3) M_{S}
$$

The Higgs fields are contained in the following representations,

$$
h_{a}^{x}=(1, \overline{2}, 2)=\left(\begin{array}{cc}
h_{2}{ }^{+} & h_{1}{ }^{0} \\
h_{2}{ }^{0} & h_{1}{ }^{-}
\end{array}\right)
$$

(where $h_{1}$ and $h_{2}$ are the low energy Higgs superfields associated with the MSSM.) The two heavy Higgs representations are

$$
H^{\alpha b}=(4,1,2)=\left(\begin{array}{cccc}
u_{H}^{R} & u_{H}^{B} & u_{H}^{G} & \nu_{H} \\
d_{H}^{R} & d_{H}^{B} & d_{H}^{G} & e_{H}^{-}
\end{array}\right)
$$

and

$$
\bar{H}_{\alpha x}=(\overline{4}, 1, \overline{2})=\left(\begin{array}{cccc}
\bar{d}_{H}^{R} & \bar{d}_{H}^{B} & \bar{d}_{H}^{G} & e_{H}^{+} \\
\bar{u}_{H}^{R} & \bar{u}_{H}^{B} & \bar{u}_{H}^{G} & \bar{\nu}_{H}
\end{array}\right) .
$$

\footnotetext{
${ }^{2}$ Note that in order to avoid anomalies of the fermionic components and preserve flatness conditions of the scalar VEVs, we introduce always $R+\bar{R}$ representations.
} 
At a scale $M_{G U T} \sim 10^{16} \mathrm{GeV}$, these Higgs fields are assumed to develop VEVs, $\langle H\rangle=$ $\left\langle\tilde{\nu}_{H}\right\rangle \sim M_{G U T}, \quad\langle\bar{H}\rangle=\left\langle\tilde{\bar{\nu}}_{H}\right\rangle \sim M_{G U T}$, leading to the symmetry breaking at $M_{G U T}$

$$
\mathrm{SU}(4) \otimes \mathrm{SU}(2)_{L} \otimes \mathrm{SU}(2)_{R} \longrightarrow \mathrm{SU}(3)_{C} \otimes \mathrm{SU}(2)_{L} \otimes \mathrm{U}(1)_{Y}
$$

in the usual notation.

Under the symmetry breaking eq. (15), the bidoublet Higgs field $h$ in eq. (12) splits into two Higgs doublets $h_{1}, h_{2}$ whose neutral components subsequently develop weak scale VEVs,

$$
\left\langle h_{1}^{0}\right\rangle=v_{1}, \quad\left\langle h_{2}^{0}\right\rangle=v_{2}
$$

with $\tan \beta \equiv v_{2} / v_{1}$.

In addition to the Higgs fields in eqs. (13) and (14) one introduces also an $\mathrm{SU}(4)$ sextet field $D=(6,1,1)$. Assuming now a $Z_{2}$ invariance with respect to $H(\bar{H}) \rightarrow$ $(-1) \times H(\bar{H})$, the tree level mass terms of the superpotential of the model read

$$
W=\lambda_{1}^{i j} \bar{F}_{i} F_{j} h+\lambda_{2} H H D+\lambda_{3} \bar{H} \bar{H} D+\mu h h
$$

Note that we have banned terms which might lead to unacceptably large neutrinohiggsino mixing [10]. Additional terms not included in eq. (17) may be forbidden by imposing suitable discrete or continuous symmetries, the details of which need not concern us here. The $D$ field carries colour and therefore does not develop a VEV, but the terms in eq. (17) $H H D$ and $\bar{H} \bar{H} D$ combine the colour triplet parts of $H, \bar{H}$ and $D$ into acceptable GUT-scale mass terms. When the $H$ fields attain their VEVs at $M_{G U T} \sim 10^{16} \mathrm{GeV}$, the superpotential of eq. (17) reduces to that of the MSSM augmented by neutrino masses. Below $M_{G U T}$ the part of the superpotential involving matter superfields is just

$$
W=\lambda_{U}^{i j} \bar{U}_{i} Q_{j} h_{2}+\lambda_{D}^{i j} \bar{D}_{i} Q_{j} h_{1}+\lambda_{E}^{i j} \bar{E}_{i} L_{j} h_{1}+\lambda_{N}^{i j} \bar{\nu}_{i} L_{j} h_{2}+\ldots
$$

where $Q_{j}$ and $L_{j}$ are the quark and lepton $S U(2)_{L}$ doublet superfields and $\bar{U}_{i}, \bar{D}_{i}$, $\bar{E}_{i}$ and $\bar{\nu}_{i}$ are the charge $2 / 3$ and $(-1 / 3)$ quark, the charged lepton and the neutrino $S U(2)_{L}$ singlet superfields. The Yukawa couplings in eq. (18) satisfy the boundary conditions

$$
\lambda_{1}^{i j}\left(M_{G U T}\right) \equiv \lambda_{U}^{i j}\left(M_{G U T}\right)=\lambda_{D}^{i j}\left(M_{G U T}\right)=\lambda_{E}^{i j}\left(M_{G U T}\right)=\lambda_{N}^{i j}\left(M_{G U T}\right) .
$$

Thus, eq. (19) retains the successful relation $m_{\tau}=m_{b}$ at $M_{G U T}$. 
The boundary conditions listed in eq. (19) lead to unacceptable mass relations for the light two families. In addition, the large family hierarchy in the Yukawa couplings appears to be unnatural since one would naively expect the dimensionless couplings all to be of the same order. This leads us to the conclusion that the $\lambda_{1}^{i j}$ in eq. (17) may not originate from the usual renormalisable tree level dimensionless coupling. The $U(1)_{X}$ symmetry will allow a renormalisable Yukawa coupling in the 33-term only and generate the rest of the effective Yukawa couplings by non-renormalisable operators that are suppressed by some higher mass scale. This suppression provides an explanation for the observed fermion mass hierarchy and occurs as powers of two expansion parameters $\epsilon$ and $\delta$ defined as follows

$$
\epsilon \equiv\langle\theta\rangle / M_{S} \sim\langle\bar{\theta}\rangle / M_{S} \sim M_{X} / M_{S}, \quad \delta \equiv\langle H\rangle\langle\bar{H}\rangle / M_{Y}^{2}=M_{G U T}^{2} / M_{Y}^{2}
$$

where $M_{X} \sim\langle\theta\rangle \sim\langle\bar{\theta}\rangle$ is the $U(1)_{X}$ symmetry breaking scale and $M_{Y}$ is a heavy mass which should be of order $M_{X}$ if the operators have a field theoretic origin, or $M_{S}$ if the operator's origin is from the string.

The $U(1)_{X}$ symmetry used is anomalous [. However, the $U(1)$ charges $Q_{X}$ are such that all of the mixed anomalies are equal, so that we can appeal to Green-Schwarz string anomaly cancellation. Moreover, the spontaneous breaking of the $U(1)_{X}$ via the $\theta, \bar{\theta}$-VEVs at a high scale should proceed along a flat direction to avoid large vacuum energy contributions from D-terms.

Using the $\theta, \bar{\theta}$ fields, we generate all effective Yukawa terms (except the (33) elements) by operators of the form

$$
O_{i j} \sim\left(\bar{F}_{i} F_{j}\right) h\left(\frac{H \bar{H}}{M_{Y}^{2}}\right)^{r}\left(\frac{\theta^{n} \bar{\theta}^{m}}{M_{S}^{n+m}}\right) \sim \bar{F}_{i} F_{j} h \delta^{r} \epsilon^{n+m},
$$

where $r, n, m \geq 0$ are set so that the operator is invariant under the $\mathrm{U}(1)_{X}$ family symmetry. The (33) operator is kept to be of the renormalisable (trilinear) form. When $H$ and $\bar{H}$ develop their VEVs, the operators in eq. (14) will become effective Yukawa couplings of the form $\bar{F} F h$ with a small coefficient of order $\left(M_{G U T}^{2} / M_{Y}^{2}\right)^{r}$. The see-saw mechanism for suppressing the mass of the three lighter neutrinos is realized in a natural way, provided that non-renormalisable terms of the form

$$
\bar{F}_{i} \bar{F}_{j}\left(\frac{H H}{M_{X}}\right)\left(\frac{\theta^{o} \bar{\theta}^{p}}{M_{S}^{o+p}}\right) \sim \bar{F}_{i} \bar{F}_{j} M_{G U T} \sqrt{\delta} \epsilon^{o+p}
$$

\footnotetext{
${ }^{3}$ In fact, in the context of string derived models, one linear combination among the various $U(1)$ symmetries accompanying the non-abelian gauge group factors, is anomalous [3, 4, 5.
} 


\begin{tabular}{ccccc}
\hline & $Q \bar{U} h_{2}$ & $Q \bar{D} h_{1}$ & $L \bar{E} h_{1}$ & $L N h_{2}$ \\
\hline$O^{A}$ & 1 & 1 & 1 & 1 \\
$O^{C}$ & $\frac{1}{\sqrt{5}}$ & $\frac{1}{\sqrt{5}}$ & $\frac{-3}{\sqrt{5}}$ & $\frac{-3}{\sqrt{5}}$ \\
$O^{M}$ & 0 & $\sqrt{2}$ & $\sqrt{2}$ & 0 \\
$O^{W}$ & 0 & $\sqrt{\frac{2}{5}}$ & $-3 \sqrt{\frac{2}{5}}$ & 0 \\
\hline
\end{tabular}

Table 1: When the Higgs fields develop their VEVs at $M_{G U T}$, the $r=1$ operators utilised lead to the effective Yukawa couplings with Clebsch - Gordon coefficients as shown. We have included the relative normalisation for each of the operators.

are included in the superpotential. It should be noted that when the exponents in eq. (22) are not integers, the corresponding element is zero due to a residual discrete symmetry. Gauge unification occurs at $M_{G U T}$, and so the gauge couplings must run together between $M_{G U T}$ and $M_{S}$. This is possible, for example, when some extra states are present in the above range [12].

\section{Textures}

In ref. [7], we showed that textures exist which fit the data and explain the presence of the zeroes through highly suppressed operators. The family mass hierarchy itself is explained through suppression via $\mathrm{U}(1)_{X}$ family symmetry: all of the fundamental Yukawa couplings are of order 1 in the model. Rather than perform a complicated systematic analysis of all possible models, here we simply examine the predictions for the neutrino masses and the lepton mixing of one successful model. We choose an example of a texture (model 1 of ref. [7]), that provides a very good quality of the fit of the data on charged fermion masses $\left(\chi^{2} /\right.$ d.o.f. $=0.34$ for 3 d.f. $)$. The texture is

$$
\lambda=\left(\begin{array}{ccc}
0 & O^{M}+s . d . & 0 \\
O^{M}+O^{A} & O^{W}+s . d . & 0 \\
0 & O^{C} & O_{33}
\end{array}\right)
$$

where $O_{33}$ is the renormalisable operator and s.d. stands for small sub-dominant operators which are negligible in the down quark and charged lepton sectors, but which are responsible for the charm and up quark masses. The C-G structure of the operators $O^{A, C, M, W}$ is listed in Table 1 . They are arrived at by taking different contractions of the $\mathrm{SU}(4) \otimes \mathrm{SU}(2)_{R} \otimes \mathrm{SU}(2)_{L}$ group indices [7]. Operators listed in the table are the ones which were found to be useful phenomenologically, and which are utilised in this paper. 
The $\mathrm{U}(1)_{X}$ symmetry is responsible for high powers of $\epsilon$, reproducing the approximate zeroes assumed in eq. (23). We pick an assignment of $U(1)_{X}$ charges corresponding to family dependent charges of case 2 of ref. [7]. The charge assignment for this case appears in Table Q 2, where we have added family independent components to the $\mathrm{U}(1)_{X}$ charges in order to make the mixed anomalies of $S U(4)^{2} U(1)_{X}, S U(2)_{R}^{2} U(1)_{X}$ and $S U(2)_{L}^{2} U(1)_{X}$ equal. This allows anomaly cancellation under the GSW mechanism. The operators resulting from the charge assignments in Table 2 reproduce the

\begin{tabular}{ccccccccc}
\hline$F_{1}$ & $F_{2}$ & $F_{3}$ & $\bar{F}_{1}$ & $\bar{F}_{2}$ & $\bar{F}_{3}$ & $h$ & $H$ & $\bar{H}$ \\
\hline 4 & 0 & -1 & 3 & -1 & 1 & 0 & 1 & -1 \\
\hline
\end{tabular}

Table 2: $U(1)_{X}$ charges of fields in the model.

mass/mixing hierarchies with order 1 Yukawa couplings in the charged fermion sector if $\epsilon=0.14, \delta=0.21$. We shall take these values of expansion parameters for the analysis of the neutrino masses below. If we take only the field content of Table 2, the D-flatness constraint sets the natural scale of $|\langle\theta\rangle|^{2}$ and $|\langle\bar{\theta}\rangle|^{2}$ to be of order $g_{u}^{2} M_{P l}^{2} /\left(4 \pi^{2}\right), g_{u}$ being the unified gauge coupling constant at $M_{S}$.

At $M_{G U T}$, the Yukawa matrices are of the form

$$
\lambda^{I}=\left[\begin{array}{ccc}
0 & H_{12} e^{i \phi_{12}} x_{12}^{I} & 0 \\
H_{21} x_{21}^{I} e^{i \phi_{21}}+\tilde{H}_{21} \tilde{x}_{21}^{I} e^{i \tilde{\phi}_{21}} & H_{22} x_{22}^{I} e^{i \phi_{22}} & 0 \\
0 & H_{32} x_{32}^{I} e^{i \phi_{32}} & H_{33} e^{i \phi_{33}}
\end{array}\right],
$$

where only the dominant operators are listed. The $I$ superscript labels the charge sector, $x_{i j}^{I}$ refers to the Clebsch-Gordon coefficient relevant to the charge sector $I$ in the $i j^{\text {th }}$ position, $\phi_{i j}$ are unknown phases and $H_{i j}$ is the magnitude of the effective dimensionless Yukawa coupling in the $i j^{\text {th }}$ position. In our case $H_{i j}$ represent the effective small Yukawa couplings generated by operators from eq. (21). Any sub-dominant operators that we introduce will be denoted below by a prime and it should be borne in mind that these will only affect the up-quark and Dirac neutrino matrices. Once unphysical phases have been rotated away by re-phasing the fermion fields, only one physical phase remains. The charged fermion analysis did not significantly constrain the remaining physical phase $\phi$ and for our purposes it shall be set to zero.

Table 1 displays the Clebsch-Gordon coefficients derived from the operators utilised for this model, a subset of operators used for this and other models in ref. [7]. Putting in the Clebsch - Gordon coefficients from Table 1 we arrive at the component Yukawa 
matrices, at the GUT scale of

$$
\begin{aligned}
\lambda^{U}= & \left(\begin{array}{ccc}
0 & \eta_{12} & 0 \\
H_{21}^{\prime} & H_{22}^{\prime} & 0 \\
0 & \sqrt{2} H_{32} / \sqrt{5} & H_{33}
\end{array}\right) \\
\lambda^{D}= & \left(\begin{array}{ccc}
0 & \sqrt{2} H_{12} & 0 \\
\sqrt{2} H_{21} & H_{22} / \sqrt{5} & 0 \\
0 & -\sqrt{2} H_{32} / \sqrt{5} & H_{33}
\end{array}\right) \\
\lambda^{E}= & \left(\begin{array}{ccc}
0 & \sqrt{2} H_{12} & 0 \\
\sqrt{2} H_{21} & 3 H_{22} / \sqrt{5} & 0 \\
0 & -3 \sqrt{2} H_{32} / \sqrt{5} & H_{33}
\end{array}\right) \\
\lambda_{\text {Dirac }}^{\nu}= & \left(\begin{array}{ccc}
0 & \eta_{12} & 0 \\
H_{21}^{\prime} & H_{22}^{\prime} & 0 \\
0 & -3 \sqrt{2} H_{32} / \sqrt{5} & H_{33}
\end{array}\right)
\end{aligned}
$$

where $\eta_{12}$ is expected to be generated from a higher order non-renormalisable term.

The following GUT scale input parameters minimised the fermion mass/mixing global $\chi^{2}$ for the case considered here [7]:

$$
\begin{aligned}
& H_{22}=2.88 \times 10^{-2}, \quad H_{12}=2.81 \times 10^{-3}, \quad H_{21}=1.30 \times 10^{-3} \text {, } \\
& H_{33}=1.18, \quad H_{22}^{\prime}=1.91 \times 10^{-3}, \quad H_{21}^{\prime}=1.94 \times 10^{-3} \text {. } \\
& H_{32}=7.28 \times 10^{-2}, \quad \eta_{12} \cong 10^{-3} \text {. }
\end{aligned}
$$

These values of the parameters lead to the predictions

$$
\begin{array}{cll}
\alpha_{S}\left(M_{Z}\right)=0.119, & \tan \beta=59.5, & m_{t}=175 \\
m_{d}=6.25, & m_{s}=158, \quad m_{b}=4.24 \\
m_{c}=1.30, & \left|V_{u s}\right|=0.2211, & \left|V_{u b}\right|=3.71 \times 10^{-3} \\
m_{u}=5, & \left|V_{c b}\right|=0.038, &
\end{array}
$$

where all masses are quoted in the $\overline{M S}$ renormalisation scheme, $m_{c, b, t}$ are running masses quoted in $\mathrm{GeV}$ and $m_{u, d, s}$ are evaluated at $1 \mathrm{GeV}$ and measured in units of $\mathrm{MeV}$. The predictions for $\left|V_{u s}\right|,\left|V_{u b}\right|$ and $\left|V_{c b}\right|$ are at the scale $M_{Z}$.

\section{Neutrino masses}

Up to now, we have shown that our ansatz generates successfully the quark and charged lepton mass hierarchy. In addition, the entries of the Dirac neutrino mass matrix are 
completely determined at the unification scale since they are related to those of the up-quark mass matrix:

$$
m_{D}\left(M_{G U T}\right)=\lambda_{D i r a c}^{\nu} v_{2}
$$

Thus, the overall scale of the matrix is determined by the Higgs field VEV which gives mass to the up quark, $v_{2}=\left\langle h_{2}\right\rangle$, while the Dirac Yukawa couplings can be encoded in terms of the parameters $\delta, \epsilon$ as can be seen from (28).

We turn now to the discussion of the heavy right-handed $(\mathrm{RH})$ neutrino Majorana mass matrix $M_{R}$. Since the RH neutrinos are members of the multiplets $\bar{F}_{x \alpha}^{i}$ (eq. (10)) accommodating right-handed quarks and leptons, their charges are already fixed. Thus, within a given charge assignment, we have no freedom of choosing the relative orders of magnitude for the elements of $M_{R}$. The $U(1)_{X}$ charge of $H$ (opposite to the charge of $\bar{H}$ ) was not fixed by the charged fermion analysis. Once the $U(1)_{X}$ charges are chosen, the structure of $M_{R}$ is directly determined by the non-renormalisable terms of eq. (22). Therefore, the $M_{R}$ entries are also encoded in terms of the same parameters $\delta$ and $\epsilon$ up to Yukawa coefficients of order 1. On the other hand, the overall scale is related to the unification mass $M_{G U T}$. When the charges from Table 2 are substituted into eq. (22), it is found that the effective RH neutrino Majorana mass matrix is

$$
M_{R}=M_{G U T} \sqrt{\delta}\left(\begin{array}{ccc}
a \cdot \epsilon^{8} & \epsilon^{4} & d \cdot \epsilon^{6} \\
\epsilon^{4} & b & e \cdot \epsilon^{2} \\
d \cdot \epsilon^{6} & e \cdot \epsilon^{2} & c \cdot \epsilon^{4}
\end{array}\right)
$$

In eq. (32), $a, b, c, d, e$ are the dimensionless Yukawa couplings, mentioned above. In the case of the string derived version of the model, they are calculable and determined in terms of the gauge coupling at the unification scale. In the field theory version, however, they cannot be determined, so they will be treated as free real parameters. The suppression factors appearing in the heavy $\mathrm{RH}$ neutrino Majorana mass matrix will finally give $M_{R}<M_{G U T}$. This implies that the light neutrino masses which will be obtained through the see-saw mechanism, will turn out to be larger than might be naively expected for $M_{R} \sim M_{G U T}$.

In the following, we will show that four of the five hybrid solutions found in ref. [1] can be obtained for natural values of $a, b, c, d, e$. We will describe first the procedure of our analysis. As has already been explained, the neutrino Dirac mass matrix is completely set by the charged fermion data. A decisive role is played by the involved Yukawa couplings at the GUT scale. Since these are related to the up-quark couplings, they are obtained by using the 3-loop QCD $\otimes 1$-loop QED- $\beta$ functions below $m_{t}$, and the 


\begin{tabular}{|c|c|c|c|c|c|c|c|c|c|}
\hline & $\mathrm{a}$ & $\mathrm{b}$ & c & d & e & $\Delta m_{31}^{2}$ & $\Delta m_{21}^{2}$ & $\sin ^{2} 2 \theta_{13}$ & $\sin ^{2} 2 \theta_{12}$ \\
\hline $\mathrm{A}$ & 1.0 & 0.25 & 0.11 & 0.125 & 0.25 & $1.210^{-4}$ & $5.510^{-12}$ & $2.510^{-3}$ & 1.00 \\
\hline B & 0.75 & 0.625 & 0.407 & 1.2 & 0.457 & $9.610^{-6}$ & $8.710^{-11}$ & $3.810^{-3}$ & 0.25 \\
\hline $\mathrm{C}$ & 1.0 & 0.345 & 0.7 & 2.0 & 0.3 & $1.410^{-5}$ & $1.210^{-10}$ & $1.610^{-3}$ & 0.80 \\
\hline $\mathrm{D}$ & 1.7 & 0.25 & 0.24 & 1.91 & 0.4 & $110^{-4}$ & $3.910^{-11}$ & $3.010^{-4}$ & 1.00 \\
\hline
\end{tabular}

Table 3: Examples of values of $\Delta m_{31}^{2}, \Delta m_{21}^{2}, \sin ^{2} 2 \theta_{13}$ and $\sin ^{2} 2 \theta_{12}$ obtained in the model discussed by us and corresponding to the hybrid MSW + VO solutions A, B, C and D found in ref. [1] $\left(\Delta m_{i j}^{2}\right.$ are in units of $\left.\mathrm{eV}^{2}\right)$. The dimensionless real parameters a,b,c,d and e from the heavy $\mathrm{RH}$ neutrino Majorana mass matrix are supposed to take values in the interval $(0.1-2.0)$.

1-loop MSSM $\beta$-functions between $m_{t}$ and $M_{G U T}$, as in ref. [7]. The renormalisation effect of the Dirac Yukawa couplings of the neutrinos is negligible because they are integrated out at the heavy Majorana mass scale. Only the $\tau$-neutrino Dirac coupling is large enough to affect the results, and this is integrated out within a couple of orders of magnitude below $M_{G U T}$, i.e, at the scale where the see-saw mechanism is expected to operate. The effective light left-handed ( $\mathrm{LH})$ neutrino Majorana mass matrix at $M_{G U T}$ is then

$$
m_{\nu_{L}}^{e f f} \equiv-m_{D}^{T} M^{-1} m_{D}
$$

The light neutrino masses are given by

$$
\mathcal{O} m_{\nu_{L}}^{\text {eff }} \mathcal{O}^{T}=\left(\begin{array}{ccc}
m_{1} & 0 & 0 \\
0 & m_{2} & 0 \\
0 & 0 & m_{3}
\end{array}\right)
$$

where $\mathcal{O}$ is an orthogonal matrix that diagonalises $m_{\nu_{L}}^{\text {eff }}$ and $m_{i}$ are the light neutrino masses $\left(m_{1} \ll m_{2} \ll m_{3}\right)$. The lepton mixing matrix is calculated by $U=\mathcal{O} V_{E_{L}}$, where the matrix $V_{E_{L}}$ appears as a result of the diagonalisation of the charged lepton mass matrix. The mixing elements and $m_{1,2,3}$ are then renormalised from $M_{G U T}$ down to $m_{t}$, again using the 1-loop MSSM RGEs [1]. The renormalisation of mixing angles and masses between $m_{t}$ and $M_{Z}$ is small and neglected.

Our string motivated criteria for the naturalness of the Yukawa couplings $a, b, c, d, e$, is that they lie in the range $0.1-2.0$. By varying $a, b, c, d, e$, four predictions corresponding to hybrid oscillation solutions A, B, C, D were found that were consistent with this constraint. These are summarized in Table 3 and should be compared with the regions shown in Figs. 1 a, 1 b and 11c.

The solutions in Table 3 correspond to the following $M_{G U T}$, masses of the heavy (approximately $\mathrm{RH}$ ) neutrinos $M_{R j}^{D}, j=1,2,3$, and lepton mixing matrices (defined in 

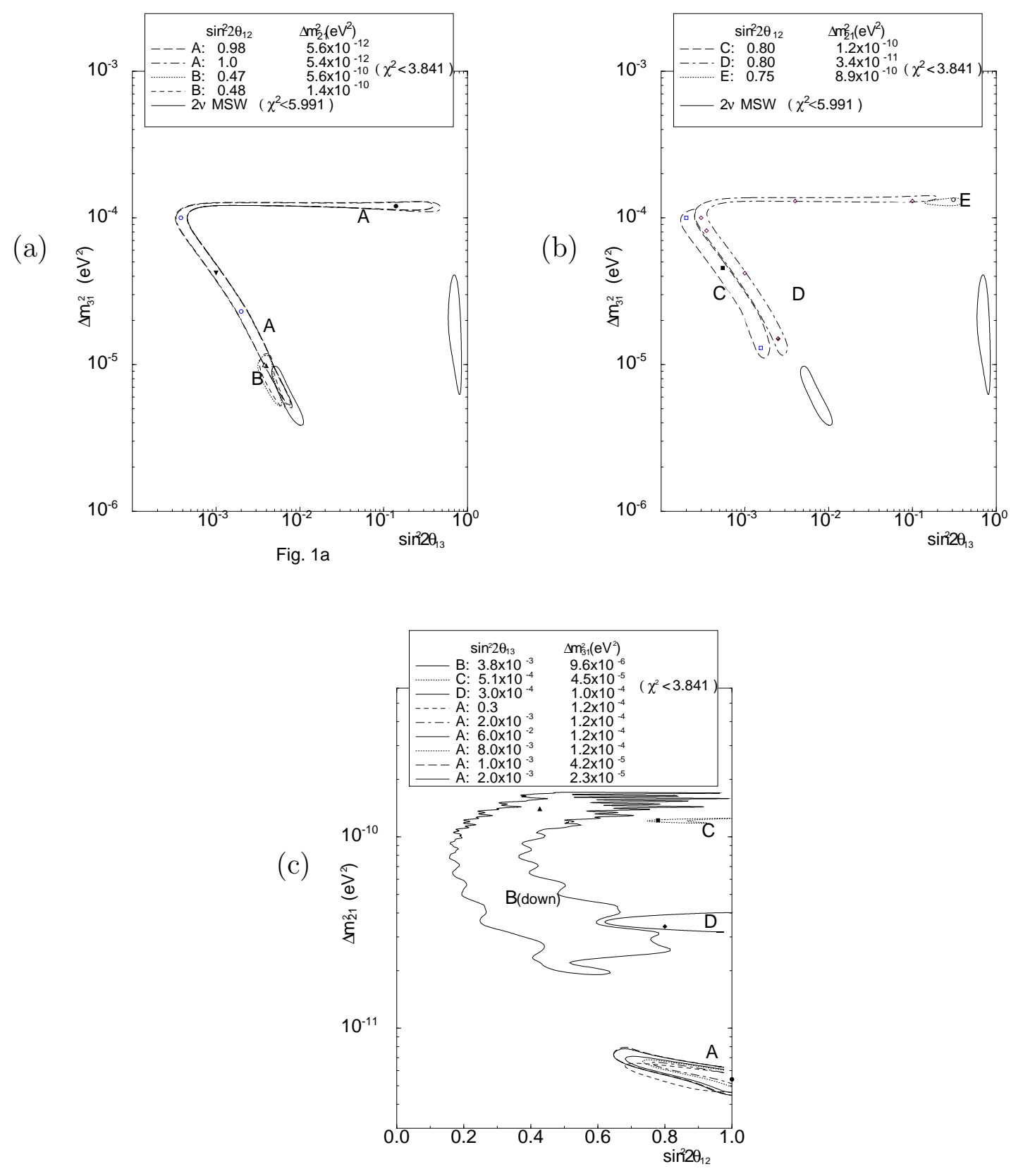

Figure 1: MSW + VO solutions of the solar neutrino problem [1] - regions of values of the MSW transition and vacuum oscillation parameters. (a) and (b) show $\Delta m_{31}^{2}$ and $\sin ^{2} 2 \theta_{13}$, corresponding to solutions A,B,C,D and $\mathrm{E}$ and obtained for fixed values of the vacuum oscillation parameters $\Delta m_{21}^{2}$ and $\sin ^{2} 2 \theta_{12}$ indicated in the figures. Shown are also the $95 \%$ C.L. $2 \nu$ MSW non-adiabatic and adiabatic solution regions. (c) shows $\Delta m_{21}^{2}$ and $\sin ^{2} 2 \theta_{12}$ for solutions $\mathrm{A}, \mathrm{B}, \mathrm{C}$, and $\mathrm{D}$. The regions correspond to fixed values of the MSW parameters $\Delta m_{31}^{2}$ and $\sin ^{2} 2 \theta_{13}$ indicated in the figure. 
the basis $\left.\left(\nu_{1}, \nu_{2}, \nu_{3}\right), m_{1}<m_{2}<m_{3}\right)$ :

$$
\begin{array}{ll}
\text { Solution A : } & M_{G U T}=0.9 \times 10^{16}, M_{R}^{D}=\left(6.8 \times 10^{14}, 4.1 \times 10^{7}, 3.0 \times 10^{13}\right) \\
& U^{A} \cong\left(\begin{array}{ccc}
0.69 & -0.72 & -0.025 \\
-0.72 & -0.69 & 0.038 \\
-0.045 & -0.0080 & -1.00
\end{array}\right) \\
\text { Solution B : } & M_{G U T}=0.8 \times 10^{16}, M_{R}^{D}=\left(2.9 \times 10^{15}, 7.7 \times 10^{7}, 1.5 \times 10^{13}\right) \\
& U^{B} \cong\left(\begin{array}{ccc}
-0.26 & 0.96 & 0.031 \\
0.96 & 0.26 & 0.057 \\
0.047 & 0.044 & -1.00
\end{array}\right) \\
\text { Solution C : } & M_{G U T}=2.5 \times 10^{16}, M_{R}^{D}=\left(1.4 \times 10^{15}, 3.0 \times 10^{7}, 7.2 \times 10^{12}\right) \\
& U^{C} \cong\left(\begin{array}{ccc}
-0.52 & 0.85 & 0.020 \\
0.85 & 0.52 & 0.066 \\
0.046 & 0.052 & -1.00
\end{array}\right) \\
\text { Solution D : } & M_{G U T}=1.9 \times 10^{16}, M_{R}^{D}=\left(6.8 \times 10^{14}, 1.4 \times 10^{7}, 1.8 \times 10^{13}\right) \\
& U^{D} \cong\left(\begin{array}{ccc}
-0.69 & 0.72 & -0.0086 \\
0.72 & 0.69 & 0.062 \\
0.051 & 0.037 & -1.00
\end{array}\right)
\end{array}
$$

where the $M_{G U T, R}$ masses are in $\mathrm{GeV}$.

The MSW + VO solutions A, B, C, and D of the solar neutrino problem of interest will be tested in the currently operating Super-Kamiokande experiment as well as in the future solar neutrino experiments SNO, BOREXINO, HELLAZ, etc. As discussed in [1], one of the distinctive predictions of the indicated hybrid MSW + VO solutions is the existence of strong and very characteristic distortions of the spectrum of ${ }^{8} \mathrm{~B}$ neutrinos, which should be observable in the Super-Kamiokande and/or SNO experiments. The seasonal (time) variation of the ${ }^{8} \mathrm{~B},{ }^{7} \mathrm{Be}$, pp, etc. solar neutrino fluxes caused by the vacuum oscillations (see, e.g., [12] and the references quoted therein) in the case of the MSW + VO solutions, cannot exceed for given values of $\Delta m_{21}^{2}$ and $\sin ^{2} 2 \theta_{12}$, the seasonal variation caused by purely two-neutrino vacuum oscillations with the same $\Delta m_{21}^{2}$ and $\sin ^{2} 2 \theta_{12}$ f. Nevertheless, the vacuum oscillation induced seasonal variations of the ${ }^{7} \mathrm{Be}$ and ${ }^{8} \mathrm{~B}$ neutrino fluxes predicted for the $\mathrm{MSW}+\mathrm{VO}$ solutions $\mathrm{B}$ and $\mathrm{C}$, can be observable in the Super-Kamiokande, SNO and BOREXINO experiments for certain regions of the solution values of the parameters [1]. For solutions A and D this effect will

\footnotetext{
${ }^{4}$ The solar neutrino flux at the Earth surface changes with the time of the year due to the ellipticity of the Earth orbit around the Sun. The change of the flux from December to June due to the standard geometrical effect is $6.68 \%$.

${ }^{5}$ This result follows directly from eq. (9) or eq. (17) in ref. [1].
} 
be observable for the ${ }^{7} \mathrm{Be}$ and $p p$-neutrinos [13], if the $p p$-neutrino flux is measured with detectors like HELLAZ or HERON. Finally, for the MSW + VO solution parameters we have obtained in the model studied here, the day-night asymmetry caused by $\nu_{e}$ and $\nu_{\mu(\tau)}$ MSW transitions in the Earth is estimated to be rather small in the signals of the detectors sensitive only to ${ }^{8} \mathrm{~B}$ or ${ }^{7} \mathrm{Be}$ neutrinos [1, 14].

\section{Conclusions}

In the present paper we have investigated the possibility to accommodate the hybrid MSW + VO solutions of the solar neutrino problem, found recently in [1], in a string motivated partially unified model. The model is based on the $S U(4) \otimes O(4)$ gauge group and was shown to provide successful predictions in the charged fermion mass sector and fit the data well. In addition, a $U(1)_{X}$ family symmetry allows the generation of effective Yukawa couplings which are naturally small, without having to put very small fundamental Yukawa couplings in the model by hand. The charged fermion data fit constrains the Dirac neutrino mass matrix and the magnitudes of the elements of the RH-neutrino Majorana mass matrix are then set by the $U(1)_{X}$ family symmetry.

We have shown in the present work that the new hybrid MSW + VO solutions to the solar neutrino problem, found in [1], appear naturally in this rather compelling model. The horizontal $U(1)_{X}$ symmetry plays a vital role in suppressing certain elements of the RH-neutrino Majorana mass matrix, allowing some of the light neutrinos to be heavier than one would be naively expecting and therefore to lie in the mass ranges required. The vertical symmetry relates the Dirac neutrino masses to the up quark masses, allowing more predictivity. This is evident in the fact that the whole $3 \times 3$ lepton mixing matrix is unambiguously determined. The predictions of the model for the neutrino masses and the lepton mixing will be tested in the currently operating and the future solar neutrino experiments (Super-Kamiokande, SNO, BOREXINO, HELLAZ, etc.).

\section{Acknowledgements}

B.C.A. would like to thank CERN for hospitality while part of this work was carried out. The work of G.K.L. is partially supported by TMR-ERBFMXR-CT96-0090. The work of S.T.P. was supported in part by the EEC grant ERBFMRX-CT96-0090 and by Grant PH-510 from the Bulgarian Science Foundation. 


\section{References}

[1] Q.Y. Liu and S. T. Petcov, Phys. Rev. D56 (1997) 7392.

[2] J. Christensen-Dalsgaard, Nucl. Phys. B (Proc. Suppl.) 48 (1996) 325; J.N. Bahcall et al., Phys. Rev. Lett. 78 (1997) 4286; S. Degl'Innocenti et al., Astr. Phys. 7 (1997) 77 and Report INFNFE - 05 -97; S. Degl'Innocenti and B. Ricci, astroph/9710292.

[3] I. Antoniadis et al., Phys. Lett. B194 (1987) 231; ibid. B231 (1989) 65.

[4] I. Antoniadis and G.K. Leontaris, Phys. Lett. B216 (1989) 333; G.K. Leontaris, ibid. B372 (1996) 212, hep-ph/9601337.

[5] A. Farragi, et al, Nucl. Phys. B335 (1990) 347; A. Farragi, Phys. Lett. B278 (1992) 131, Nucl. Phys. B403 (1993) 101; S. Chaudhuri et al., Phys. Rev. Lett. 75 (1995) 2264; Nucl. Phys. B456 (1995) 82; H.D. Dahmen et al, hep-th/9711192; D. Finnell, Phys. Rev. D53 (1996) 5781.

[6] J. Pati and A. Salam, Phys. Rev. D10 (1974) 275.

[7] B. C. Allanach et al., Phys. Rev. D56 (1997) 2632.

[8] J. Pantaleone, Phys. Rev. D43 (1991) 641.

[9] See, e.g., L. Ibanez, G.G. Ross, Phys. Lett. B332 (1994) 100; P. Binetruy and P. Ramond, ibid. B350 (1995) 49; V. Jain and R. Shrock, ibid. B352 (1995) 83.

[10] A.E. Faraggi and J.C. Pati, Phys. Lett. B400 (1997) 314.

[11] B.C. Allanach and S.F. King, Nucl. Phys. B459 (1996) 75 and Phys. Lett. B353 (1995) 477.

[12] S.M. Bilenky and B. Pontecorvo, Phys. Rep. 41 (1978) 225; P.I. Krastev and S.T. Petcov, Nucl. Phys. B449 (1995) 605.

[13] P.I. Krastev and S.T. Petcov, Phys. Rev. D53 (1996) 1665.

[14] M. Maris and S.T. Petcov, Phys. Rev. D56 (1997) 7444. 